\title{
NOTÍCIAS E COMENTÁRIOS
}

CORRESPONDÊNCIA. Esta seção de Arquivos de Neuro-Psiquiatria divulga opiniões e observações construtivas acerca de artigos publicados em seus últimos números. São aceitas cartas assinadas, sucintas e desprovidas de caráter pessoal, abordando aspectos objetivos dos artigos e utilizando até cinco referências bibliográficas.

Enviar sua contribuição em uma via acompanhada do respectivo disquete. Utilizar o endereço: Arquivos de Neuro-Psiquiatria, Praça Amadeu Amaral 47 / 33, 01327-010 São Paulo SP.

PRÊMIO DE INCLUSÃO SOCIAL DE PACIENTES ESQUIZOFRÊNICOS E BIPOLARES. A Associação Brasileira de Psiquiatria e a indústria farmacêutica Eli Lilly do Brasil estão lançando este prêmio que tem por objetivo incentivar programas focados na reintegração social de tais pacientes. Este prêmio é nacional e abrange três categorias. Além de diploma e troféu, o vencedor em cada categoria vai receber $\mathrm{R} \$ 20000$. Informações sobre como concorrer estão sendo fornecidas (www.premiodeinclusao.com.br) ou podem ser obtidas (e-mail organizacao@premiodeinclusao. com.br). Inscrições: até 31 julho. Julgamento: três diferentes comissões de alto nível analisarão os trabaIhos inscritos, sendo indicados os vencedores. Entrega do Prêmio: entre 15 e 18 outubro. Outros informes: Regina C Rocha de Cunto, Rumo Comunicação Empresarial (Fone 113742 3601).

LIGA BRASILEIRA DE EPILEPSIA (LBE) / CAPÍTULO PAULISTA. De 12 a 15 junho 2003 em Campinas realiza-se o Encontro da Campanha Global, organizado pelo colega Li Li Min. O Evento do Capítulo Paulista da LBE foi transferido para 9 e 10 agosto 2003 em São Paulo (Hotel Transamérica, Alameda Santos 981), organizado pela Diretoria do Capítulo, que tem à frente a colega Maria Luíza Giraldes Manreza. Informes e inscrições: $L B E$, Rua Teodoro Sampaio 741 / 94, 05405-050 São Paulo SP (Fax 11 3085 6574.E-mail lbe-br@uol.com.br). de São Paulo (Lorena Flat Hotel, Avenida Rebouças 455), têm continuação estes cursos ministrados pelo colega Ciro da Silva, da Universidade de São Paulo, patrocinados por TRB Pharma (e-mail: trb@trbpharma.com.br). O primeiro módulo desse curso foi realizado em $15 \mathrm{e}$ 16 março 2003 (XXIV Curso Intensivo de Anatomia Topográfica e Seccional do Sistema Nervoso Aplicada à Neuroimagem). O segundo módulo, iniciado em 26 abril e continuado em 25 maio (XVI Curso Anual de Anatomia Funcional do Sistema Nervoso Humano), se encerra em 28 e 29 junho, com a apresentação dos temas: conexões e funções do cerebelo e dos sitemas vestibular e auditivo, conexões e funções do sistema límbico, anatomia seccional das fossas cranianas anterior, média e posterior (correlações). O terceiro módulo (II Curso Intensivo de Anatomia Topográfica e Seccional da Fossa Craniana Posterior Aplicada à Neuroimagem) está programado para 27 e 28 setembro e abrange os temas: anatomia topográfica do tronco encefálico e do cerebelo, sistematização das vias ascendentes e descendentes do tronco encefálico, estrutura e funções das porções periférica e central dos nervos cranianos, anatomia topográfica dos nervos cranianos, correlação entre anatomia seccional e imagem (planos axial, plano sagital e coronal). Informes e inscrições: Sra. Carolina Dias, Fone 1191359611.

REUNIÕES DE DISCUSSÃO DE CASOS DE NEUROLOGIA INFANTIL. Em 17 março 2003 teve lugar a 45a reunião na Faculdade de Ciências Médicas a Santa Casa de Misericórdia de São Paulo (FCM), supervisionada pelos colegas Sérgio Rosemberg e Fernando $\mathrm{N}$ Arida, com a participação ativa de médicos residentes e estagiários em neurologia infantil da FCM, UNFESP, USP e UNICAMP. Em junho 2003 na FMUSP (Hospital das Clínicas) realiza-se a $46^{\mathrm{a}}$ reunião, supervisionada pela colega Umbertina Conti Reed. Informes e inscrições: Serviço de Neurologia Infantil, Hospital das Clínicas FMUSP, Avenida Enéas CarvaIho Aguiar, São Paulo SP (Fax 113062 0063). 
PSIQUIATRIA INFANTIL. De 26 a 28 junho 2003 em Vitória ES (Centro de Convenções) realiza-se este tradicional evento da Associação Brasileira de Neurologia e Psiquiatria Infantil (ABNEPI). Tema central: saúde mental na infância e adolescência: diagnóstico e tratamento. Informes e inscrições: PROVEN Eventos Ltda, Avenida Nossa Senhora da Penha 565 / 111, 29055-903 Vitória ES (Fax 273315 7101. Email cbnpi@terra.com.br).

$3^{\circ}$ ENCONTRO DA SOCIEDADE BRASILEIRA DE INVESTIGAÇÃO NEUROLÓGICA (SBIN). Em 15 e 16 agosto 2003, em Curitiba (Hotel Four Points Sheraton) realiza-se este evento anual da SBIN, presidido pelo colega Lineu César Werneck. Inscrição de temas: até $\mathbf{3 0}$ junho. Informes e inscrições: SBIN (www.sbin.org.br), Rua Ébano Pereira 60 / 1905, 80240-902 Curitiba PR (Fax 412333092 / 2224211. E-mail: sbin@sbin.org.br).

SIMPÓSIO INTERNACIONAL SOBRE TRANSTORNOS DO HUMOR - 20 ANOS DO GRUDA. Em 15 e 16 agosto 2003 na cidade de São Paulo (Centro de Convenções Pompéia) realiza-se este evento, comemorando os vinte anos do GRUDA (Grupo de Estudos de Doenças Afetivas) do Instituto de Psiquiatria do Hospital das Clínicas da Faculdade de Medicina da Universidade de São Paulo. O simpósio tem como coordenadores os colegas Ricardo Alberto Moreno e Beny Lafer, membros do GRUDA. Informes e inscrições: BL Congressos e Eventos, Rua Ana Luísa de Souza 197, 03882-170 São Paulo SP (Fax 116146 0314.E-mail bleventos@uol.com.br).

I CONGRESSO BRASILEIRO DE NEUROLOGIA INFANTIL, VIII ENCONTRO DA SOCIEDADE BRASILEIRA DE NEUROLOGIA INFANTIL (SBNI), I ENCONTRO INTERNACIONAL DE NEURORRADIOLOGIA PEDIÁTRICA DA SBNRID. De 15 a 17 agosto 2003 na cidade de São Paulo (Hotel Blue Tree). Este evento tem Fernando Kok como Presidente e Fernando Arida como Secretário Geral. Temas: neuroimagem, fronteiras entre a neurologia e a psiquiatria infantil terapêutica em neurologia infantil. Informes e inscrições: www.eventus.com.br/sbni (Fax 113361 3089. Email: eventus@eventus.com.br).
SIMPÓSIO INTERNACIONAL DE NEUROLOGIA NEONATAL E NEONATOLOGIA. De 21 a 23 agosto 2003 em Curitiba. Informes com o colega Sérgio Antoniuk (Fones (41) 223 2570, 3222208 e 254 3221.E-mail:antoniuk@uol.com.brou acdefarias@ uol.com.br).

XVII CONGRESSO BRASILEIRO DE CEFALÉIA. De 21 a 23 agosto 2003 na cidade do Recife (Mar Hotel). Este congresso é patrocinado pela Sociedade Brasileira de Cefaléia (SBC), cujo Presidente é o colega Pedro Ferreira Moreira Filho. Ele e o colega Wilson Farias da Silva presidem a Comissão Organizadora deste evento. Inscrição de temas livres: até 13 junho 2003. Informes e inscrições: JZ Congressos (www.jz.com.br), Rua Conde de Irajá $260 / 2^{\circ}$ andar, 22271-020 Rio de Janeiro RJ (Fax 212537 9134. Email: cefaleia@jz.com.br).

$7^{\text {th }}$ CONGRESS OF THE EUROPEAN FEDERATION OF NEUROLOGICAL SOCIETIES (EFNS 2003). De 30 agosto a 2 setembro 2003, em Helsinki (Helsinki Fair Centre) realiza-se este evento da EFNS (www.efns.org), recebido pela Finish Neurological Society e copatrocinado pela European Section of the Movement Disorders Society, tendo Jacques L. De Reuck à frente do Congress Programme Committee. Informes e inscrições: Kenes International (www.kenes.com/efns2003), 17 rue du Cendrier, $\mathrm{CH}$ 1211, Geneva 1, Switzerland (Fax 41227322850. E-mail: efns03@kenes.com).

III ENCONTRO BRASILEIRO DE DISTÚRBIOS DO MOVIMENTO. De 12 a 13 de setembro 2003, em Foz do Iguaçú (Hotel Mabú). Comissão Organizadora: Hélio A.G. Teive (HC-UFPR), Francisco E.C. Cardoso (HC-UFMG), Egberto Reis Barbosa (HC-FMUSP) e Luiz Augusto Franco de Andrade (Hospital do Servidor Público do Estado de São Paulo). Informações: Idealiza Eventos (Fax 41242 7175; www. idealiza.com.br; e-mail: idealiza@idealiza.com.br)

$1{ }^{\circ}$ CONGRESSO GOIANO DE NEUROLOGIA. De 18 a 20 setembro 2003, em Goiânia (Castro's Park Hotel). Este congresso é patrocinado pela Sociedade de Neurologia do Estado de Goiás (SONG), cujo 
Presidente é colega Sebastião Eurico de Melo Souza. Informes e inscrições: Classe Promoções e Eventos (Fax 622240733 / 2830. E-mail: classeeventos@ classeeventos.com.br).

XI CONGRESSO PANAMERICANO DE NEUROLOGIA \& 58 CONGRESO CHILENO DE NEUROLOGÍA, PSIQUIATRÍA y NEUROCIRUGÍA. De 8 a 12 outubro 2003 em Santiago, Chile (Centro de Convenciones Diego Portales), presidido pelo colega Pedro Chamá e tendo como representante da Federação Mundial de Neurologia o colega Jorge Tapia. Temas oficiais de neurologia: sono, cefaléias, demências, doença cerebrovascular, doenças do sistema nervoso autônomo, epilepsia, esclerose múltipla, miopatias, nervo periférico, neuroepidemiologia, neurofisiologia, neuroftalmologia, neurogenética, neuroinfectologia, neurointensivismo, neuroncologia, neurotologia, transtornos do movimento, educação. Temas oficiais de psiquiatria: corrupção, dependências, esquizofrenia, guias clínicos, psiconeuroendocrinologia, psiquiatria do trabalho, sexualidade, $T A B$, transtornos da personalidade, plano nacional de antipsicóticos. Temas oficiais de neurocirurgia: neurocirurgia vascular, neurocirurgia de tumores, neurocirurgia de nervo periférico, neurocirurgia pediátrica. Temas livres: inscrições até 15 junho 2003 (postal) e até $\mathbf{3 0}$ junho 2003 (internet). Secretaria Científica (www.sonepsyn.cl): Sra. Susana Salvadores (Fax 562 234 2460; e-mail: sonepsym. secgeneral@terra.cl). Informes e inscrições na Secretaria : Trajamar Congresos \& Eventos [Sra. Luisa Martínez (Imartinez @tajamar.cl) e Srta. Julieta Martínez (jmartinez@ tajamar.cl). Fax 562233 2996]. Outros dados se encontram disponíveis no site do congresso (www. panamerica noneurologia.cl).

IV CONGRESSO BRASILEIRO DE DOENÇAS CEREBROVASCULARES E VIII JORNADA SUL BRASILEIRA DE NEUROLOGIA E NEUROCIRURGIA. De 16 a 19 outubro 2003 em Porto Alegre (Centro de Eventos Hotel Plaza São Rafael).Estes dois eventos se realizam conjugatamente. O primeiro é patrocinado pela Sociedade Brasileira de Doenças Cerebrovasculares (SBDCV) e tem como Presidente o colega Sérgio Roberto Haussen. O segundo tem como Presidente a colega Liselotte Menke Barea. Temas Livres: inscrição até 15 julho 2003. Informes e inscrições: Plenarium Organização de Eventos, Rua Ramiro Barcelos 820, 90035-001 Porto Alegre Rs (Fax 5133118969 /9456.E-mail plenar@terra.com.).

$27^{\text {th }}$ INTERNATIONAL CONGRESS OF CLINICAL NEUROPHYSIOLOGY. De 16 a 20 setembro 2003 em San Francisco CA (Hilton Towers) preparado pela American Association of Electrodiagnostic Medicine (AAEM) com a American Clinical Physiology Society e a International Society for Transcranial Stimulation. Informes e inscrições: AAEM (www.aaem.net), 421 First Avenue SW suite 300E, Rochester MN 55902 USA (Fax 1507288 1225. E-mail aaem@aaem.net).

AMERICAN NEUROLOGICAL ASSOCIATION (ANA): $128^{\text {th }}$ ANNUAL MEETING. De 19 a 22 outubro 2003 em San Francisco (Marriott Hotel). Informes e inscrições: ANA Executive Office (www.aneuroa.org; email anameeting@llmsi.com).

XIX CONGRESSO BRASILEIRO DE NEUROFISIOLOGIA CLÍNICA, XXVII REUNIÃO DA LIGA BRASILEIRA DE EPILEPSIA E SIMPÓSIO DE SONO DO CAPÍTULO LATINO-AMERICANO DA IFCN. De 22 a 25 outubro 2003 na cidade do Rio de Janeiro (Hotel Sofitel) , realizado pela Sociedade Brasileira de Neurofisiologia Clínica (www.neurofisio.com.br). A Comissão Organizadora tem como Presidente a colega Márcia Waddington Cruz. Temas Livres: inscrição até $\mathbf{3 1}$ julho. Informes e inscrições: Multitravel Turismo e Eventos, Rua Visconde de Pirajá 550 / 2015, 22410-002 Rio de Janeiro RJ (Fax 212540 8629. E-mailmt.neurofisio@uol.com.br).

$7^{\text {th }}$ CONGRESS OF WORLD FEDERATION OF INTERVENTIONAL AND THERAPEUTIC NEURORADIOLOGY (WFITN 2003). De 2 a 5 novembro 2003 na cidade do Recife (Summerville Beach Resort). Outras informações: www.wfitn2003.org.br

$X$ CONGRESSO DA ACADEMIA BRASILEIRA DE NEUROCIRURGIA. De 5 a 8 novembro 2003 na cidade do Recife (Praia do Muro Alto, Porto de Galinhas), precedido de 2 a 4 novembro 2003 pelo Curso de Educação Continuada da World Federation of 
Neurosurgical Societies. Em 5 novembro, conjuntamente com o Congresso Mundial da World Federation of Interventional and Therapeutic Neuroradiology (notícia acima), será realizada reunião para discussão de patologia vascular entre os neurocirurgiões endovasculares. Todos os detalhes sobre o congresso estão disponíves em seu site (www.abnc2003. com.br).

INTERNATIONAL COLLOQUIUM ON CEREBROSPINAL FLUID ANALYSIS IN TROPICAL NEUROLOGY. De 26 a 29 novembro 2003 em Antuérpia (Prince Leopold Institute of Tropical Neurology) realiza-se este evento. O Comitê de Organização tem à frente V. Lejon. Informes e inscrições (www.itg.becolloq2003) Danielle van Melle, Institute of Tropical Medicine, Nationalestraat 155, 2000 Antwerp, Belgium (Fax 323247 6213.E-mail dvmelle@itg.be).

ISI THOMSON NEWS RELEASE. March 3 anuncia o acesso direto ao Patent Documents Worldwide via the Thomson Patent Store; March 17 informa sobre Derwent Innovations Index Enhanced With Expanded Chemistry Content and Search Functionality; April 8 informa sobr Thomson Derwent Launches Patent ProfilesNet; April 21 informa sobre o Australian ViceChancellors' Committee Consortium Increases Access to ISI Web of Science Content. Para detalhes, dirigirse a: Rodney Yancey (rodney.yancey@ isinet.com) ou a: ISI (www.isinet.com), 3501 Market Street, Philadelphia, PA 19104 USA (Fax 1215387 4706).

BIOSIS News Release. January 10 noticia a a introdução do BiologyBrowser, um portal interativo de Life Sciences, January $\mathbf{2 4}$ informa que o Full File of BIOSIS Previews passa a estar disponível no sistema EBSCOhost.Para detalhes, contatuar: Marisa Westcott (mwescott@biosis.org) ou consultarBIOSIS (www.biosis.org),Two Commerce Square, 2001 Markedt Street suite 700, Philadelphia, PA 191037095 USA (Fax 1215587 2016.E-mail info@ mail.biosis.org). 